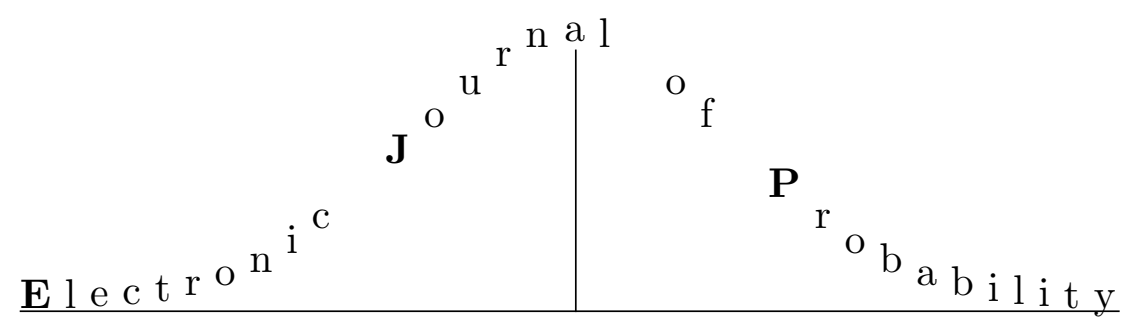

Vol. 15 (2010), Paper no. 50, pages 1556-1573.

Journal URL

http://www.math.washington.edu/ ejpecp/

\title{
Exponential Estimates for Stochastic Convolutions in 2-smooth Banach Spaces
}

\author{
Jan Seidler \\ Institute of Information Theory and Automation \\ Academy of Science of the Czech Republic \\ Pod vodárenskou věží 4 \\ 18208 Praha 8, Czech Republic \\ seidler@utia.cas.cz \\ http://simu0292.utia.cas.cz/seidler
}

\begin{abstract}
.
Sharp constants in a (one-sided) Burkholder-Davis-Gundy type estimate for stochastic integrals in a 2-smooth Banach space are found. As a consequence, exponential tail estimates for stochastic convolutions are obtained via Zygmund's extrapolation theorem.

Key words: stochastic integrals in 2-smooth Banach spaces, BurkholderDavis-Gundy inequality, exponential tail estimates, stochastic convolutions. AMS 2000 Classification: $60 \mathrm{H} 15$.

Submitted to EJP on April 13, 2010, final version accepted September 6, 2010.
\end{abstract}

This research was supported by the GA ČR Grant No. 201/07/0237. 
0. Introduction. The role played by stochastic convolutions in the semigroup theory of (semilinear) stochastic partial differential equations is well understood nowadays, see for example the monographs [7] or [6] for a systematic exposition. If $E$ and $H$ are separable Hilbert spaces, $W$ a (cylindrical) Wiener process on $H$, $\left(e^{A t}\right)$ a $C_{0}$-semigroup on $E$ and $\psi$ a progressively measurable process with values in a suitable space of linear operators from $H$ to $E$, then the stochastic convolution is a random process of the form

$$
J_{t}(\psi)=\int_{0}^{t} e^{A(t-s)} \psi(s) \mathrm{d} W(s), \quad t \geq 0 .
$$

Unfortunately, $J(\psi)$ is a not a (local) martingale, and its basic properties are far from being obvious. In particular, to establish $L^{p}$-estimates for $J(\psi)$ that would replace martingale moment inequalities which are no longer available requires new ideas. One may consult the above quoted books and references therein to get acquainted with methods developed to surmount this problem.

In the paper [24] we showed that $L^{p}$-estimates of $J(\psi)$ with sharp constants (that is, constants which grow as $p^{1 / 2}$ as $p \rightarrow \infty$ ) lead in a very simple way, via Zygmund's extrapolation theorem, to exponential tail estimates

$$
\mathrm{P}\left\{\sup _{0 \leq t \leq T}\left\|\int_{0}^{t} e^{A(t-s)} \psi_{s} \mathrm{~d} W_{s}\right\| \geq \varepsilon\right\} \leq k_{1} e^{-k_{2} \varepsilon^{2}}, \quad \varepsilon \geq 0 .
$$

Moreover, it was proved that the constants in $L^{p}$-estimates for $J(\psi)$ are asymptotically equivalent as $p \rightarrow \infty$ to the constants $C_{p}$ in the Burkholder-Davis-Gundy inequality

$$
\left\|\sup _{t \geq 0}\right\| M_{t}\left\|_{E}\right\|_{L^{p}(\Omega)} \leq C_{p}\left\|\langle M\rangle_{\infty}^{1 / 2}\right\|_{L^{p}(\Omega)}
$$

for $E$-valued continuous martingales. For real-valued continuous martingales at least three different proofs that

$$
C_{p}=O\left(p^{1 / 2}\right), \quad p \rightarrow \infty
$$

are available (cf. [8], Theorem 3.1; [1], Proposition 4.2; [23], Theorem 1); (0.3) may be easily extended to $E$-valued continuous martingales by using a general procedure proposed in [14] (see [24] for details).

To obtain finer regularity results for solutions to stochastic partial differential equations it is often advantageous to abandon Hilbertian setting and work in a 2-smooth Banach space $E$, typically, in $E=L^{q}(\mu)$ for some $q \geq 2$, see [3] for a pioneering work in this direction. (The first to develop stochastic integration theory in 2-smooth Banach spaces was A. Neidhardt in his Ph.D. thesis [17] which, however, has been never published. From several available expositions, we shall use the paper [18] since the theory is presented there in a form suitable for our purposes.) In a 2-smooth Banach space $E$, no estimate like (0.2) is known for general continuous 
$E$-valued martingales, nevertheless a closely related inequality may be proved in a particular case of stochastic integrals driven by a Wiener process. However, the methods used to derive (0.3) in the Hilbertian case cease to be applicable in 2 -smooth spaces.

The main result of the present paper is a proof that (0.3) remains valid for the constant $C_{p}$ appearing in the Burkholder-Gundy-Davis type inequality for stochastic integrals in a 2-smooth Banach space E. Our approach is based on the Rosenthal inequality for discrete-time martingales, which we use in a form that was obtained in [13] for real-valued martingales and in [22] for $E$-valued processes. Exponential tail estimates $(0.1)$ for stochastic convolutions in $E$ then follow in a very straightforward manner.

To state our results precisely, we have to introduce some notation and recall a few results. Hereafter, $X$ will be a separable 2 -smooth Banach space, $\Upsilon$ a separable real Hilbert space, and $W$ a cylindrical Wiener process on $\Upsilon$ with a covariance operator $Q \in \mathscr{L}(\Upsilon)$, defined on a stochastic basis $\left(\Omega, \mathscr{F},\left(\mathscr{F}_{t}\right), \mathrm{P}\right)$. Let us denote by $U$ the space $\operatorname{Rng} Q^{1 / 2}$ equipped with the norm $\|x\|_{U}=\left\|Q^{-1 / 2} x\right\|_{\Upsilon}$, where $Q^{-1 / 2}$ denotes the inverse to the restriction of the operator $Q^{1 / 2}$ to $\left(\operatorname{Ker} Q^{1 / 2}\right)^{\perp}$. The space of all $\gamma$-radonifying operators from some Hilbert space $H$ to $X$ will be denoted by $\gamma(H, X)$ and its norm by $\|\cdot\|_{\gamma(H, X)}$. We shall write simply $\|\cdot\|_{\gamma}$ instead of $\|\cdot\|_{\gamma(U, X)}$ if there is no danger of confusion. By $\Gamma(U, X)$ we shall denote the set of all progressively measurable $\gamma(U, X)$-valued stochastic processes $\psi$ satisfying $\|\psi\|_{\gamma} \in L_{\text {loc }}^{2}\left(\mathbb{R}_{+}\right)$almost surely, that is,

$$
\int_{0}^{t}\|\psi(s)\|_{\gamma}^{2} \mathrm{~d} s<\infty \quad \text { for all } t \geq 0 \text { P-almost surely. }
$$

Let $\operatorname{Fin}(\Upsilon, X)$ denote the space of all finite dimensional operators from $\Upsilon$ to $X$, i.e., $B: \Upsilon \rightarrow X$ belongs to $\operatorname{Fin}(\Upsilon, X)$ if and only if $B=\sum_{k=1}^{K} h_{k} \otimes x_{k}$ for some $h_{k} \in \Upsilon$ and $x_{k} \in X$. If $B$ has this representation then $B W_{t}$ is a well-defined random variable in $X$, namely

$$
B W_{t}=\sum_{k=1}^{K} W_{t}\left(h_{k}\right) x_{k} .
$$

We shall write $B\left(W_{t}-W_{s}\right)$ instead of $B W_{t}-B W_{s}$. We shall use repeatedly the following estimate (see e.g. [18], Lemma 3.3): for every $p>0$ there exists a constant $\alpha_{p}<\infty$ such that

$$
\mathrm{E}\left\|B\left(W_{t}-W_{s}\right)\right\|_{X}^{p} \leq \alpha_{p}^{p}(t-s)^{p / 2}\left\|B Q^{1 / 2}\right\|_{\gamma(\Upsilon, X)}^{p}
$$

for all $B \in \operatorname{Fin}(\Upsilon, X)$ and $t \geq s \geq 0$. If $p=2$ then $\alpha_{2}=1$ and equality holds in $(0.4)$.

Finally, the norm of the space $L^{p}(\Omega)$ will be denoted by $|\cdot|_{p}$.

It is known (see again e.g. [18]) that for any $\psi \in \Gamma(U, X)$ a stochastic integral

$$
I_{t}(\psi)=\int_{0}^{t} \psi(s) \mathrm{d} W(s), \quad t \geq 0
$$


is well-defined and $I_{\bullet}(\psi)$ is a local martingale in $X$ having continuous trajectories. Moreover, the following form of a (one-sided) Burkholder-Davis-Gundy inequality holds true: for any $p>0$ a constant $C_{p}<\infty$ may be found such that

$$
\mathrm{E} \sup _{0 \leq t \leq T}\left\|\int_{0}^{t} \psi_{s} \mathrm{~d} W_{s}\right\|^{p} \leq C_{p}^{p} \mathrm{E}\left(\int_{0}^{T}\left\|\psi_{s}\right\|_{\gamma}^{2} \mathrm{~d} s\right)^{p / 2}
$$

for all $T>0$ and all $\psi \in \Gamma(U, X)$. Several proofs of $(0.5)$ are available in the literature. A proof using the Itô formula is provided in [4], Theorem 3.1, under an additional hypothesis upon smoothness of the norm $\|\cdot\|_{X}$. The constant $C_{p}$ obtained in [4] depends on constants appearing in this extra hypothesis. If $X=L^{q}$, $q \geq 2$, then $C_{p}=O(p)$ as may be expected with reference to the employed method. (Note that a related result including a Burkholder-type inequality for stochastic convolutions was proved by the same argument already in [5], Theorem 1.1. The estimate (0.5) is also stated in [3], but a complete proof does not seem to be given there.) Ondreját's proof $([18], \S 5)$ is based on good $\lambda$-inequalities and, according to [19], it again leads to a constant $C_{p}=O(p), p \rightarrow \infty$. These $O(p)$-estimates are not sufficient for our purposes. The first proof of (0.5), up to our knowledge, was given by E. Dettweiler in [10], Theorem 4.2 (cf. also [9], Theorem 1.4). His proof uses the same basic tool as we do in the present paper - a martingale version of Rosenthal's inequality is applied to a discrete approximation of a stochastic integral in $X$ - and so it is possible that it may yield a constant $C_{p}$ with a desired growth. However, Dettweiler works in a rather general setting and it seems difficult to figure out a precise value of the constant he obtained. Therefore, we aim at providing an alternative proof of (0.5) leading to a constant with an optimal growth $C_{p}=O(\sqrt{p})$.

Acknowledgements. Thanks are due to Martin Ondreját who offered valuable comments on a preliminary version of this paper.

1. The MAIn RESUlt. We shall prove the following refinement of the inequality $(0.5)$ :

Theorem 1.1. Let $X$ be a separable 2-smooth Banach space, $W$ a cylindrical $Q$-Wiener process on a real separable Hilbert space $\Upsilon$ and $U=\operatorname{Rng} Q^{1 / 2}$. There exists a constant $\Pi<\infty$, dependent only on $\left(X,\|\cdot\|_{X}\right)$, such that

$$
\left.\left|\sup _{0 \leq t \leq \tau} \| \int_{0}^{t} \psi(s) \mathrm{d} W(s)\right|_{X}\right|_{p} \leq \Pi \sqrt{p}\left|\left(\int_{0}^{\tau}\|\psi(s)\|_{\gamma}^{2} \mathrm{~d} s\right)^{1 / 2}\right|_{p}
$$

holds whenever $p>2, \tau$ is a stopping time and $\psi$ is in $\Gamma(U, X)$.

The estimate $(0.5)$ for $p \in] 0,2]$ follows from (1.1) by a standard application of Lenglart's inequality. However, we shall use implicitly the $p=2$ case of $(0.5)$, since it is hidden in the construction of a stochastic integral in [18] we rely on. This might be avoided by a small modification of the proof, but this point is unimportant for the aims of our paper.

The proof of Theorem 1.1 is deferred to Section 3. 
2. Applications to stochastic convolutions. Let $\left(e^{A t}\right)$ be a $C_{0}$-semigroup on $X$, the stochastic convolution is defined by

$$
J_{t}(\psi)=\int_{0}^{t} e^{A(t-s)} \psi(s) \mathrm{d} W(s), \quad t \geq 0,
$$

for progressively measurable processes $\psi$ satisfying

$$
\int_{0}^{t}\left\|e^{A(t-s)} \psi(s)\right\|_{\gamma}^{2} \mathrm{~d} s<\infty \quad \text { for all } t \geq 0 \text { P-almost surely. }
$$

Our goal is to prove exponential $L^{2}$-integrability of $J(\psi)$ by combining Theorem 1.1 with Zygmund's extrapolation theorem.

This is rather straightforward for contractive and positive (i.e., positivity preserving) semigroups on an $L^{q}$-space, since then we may pass directly from stochastic integrals to stochastic convolutions using Fendler's theorem on isometric dilations (see [12], Section 4 , for details). Assume that $(M, \mathscr{M}, \mu)$ is a separable measure space, $q \in\left[2, \infty\left[\right.\right.$, and $X=L^{q}(\mu)$, hence $X$ is a separable 2-smooth space. Suppose further that $\left(e^{A t}\right)$ is a strongly continuous semigroup of positive contractions on $L^{q}(\mu)$. Then [12], Proposition 4.1, together with Theorem 1.1 imply that

$$
\left|\sup _{0 \leq t \leq T}\left\|J_{t}(\psi)\right\|_{L^{q}(\mu)}\right|_{p} \leq \Pi \sqrt{p}\left|\left(\int_{0}^{T}\|\psi(s)\|_{\gamma\left(U, L^{q}(\mu)\right)}^{2} \mathrm{~d} s\right)^{1 / 2}\right|_{p}
$$

holds for all $p>2, T \in \mathbb{R}_{+}$and every stochastic process $\psi \in \Gamma\left(U, L^{q}(\mu)\right), \Pi$ being the constant from Theorem 1.1. Let us fix $T>0$, denote by $\operatorname{Dom}(\boldsymbol{J})$ the cone of progressively measurable processes in $L^{p}\left(\Omega ; L^{2}\left((0, T) ; \gamma\left(U, L^{q}(\mu)\right)\right)\right)$ and define a mapping

$$
\boldsymbol{J}: \operatorname{Dom}(\boldsymbol{J}) \longrightarrow L^{p}(\Omega), \quad \psi \longmapsto \sup _{0 \leq t \leq T}\left\|J_{t}(\psi)\right\|_{L^{q}(\mu)} .
$$

The mapping $\boldsymbol{J}$ is sublinear, positive homogeneous and, by (2.2), satisfies

$$
\|\boldsymbol{J}(\psi)\|_{L^{p}(\Omega)} \leq \Pi \sqrt{p}\|\psi\|_{L^{p}\left(\Omega ; L^{2}\left((0, T) ; \gamma\left(U, L^{q}(\mu)\right)\right)\right)}, \quad p>2 .
$$

From Zygmund's theorem (see [26], Theorem XII.4.41; the easy extension to vectorvalued functions may be found in [24], Theorem A.1) we get the following result:

Theorem 2.1. Let $(M, \mathscr{M}, \mu)$ be a separable measure space, $q \in\left[2, \infty\left[, T \in \mathbb{R}_{+}\right.\right.$, and $\left(e^{A t}\right)$ a positive contractive $C_{0}$-semigroup on $L^{q}(\mu)$. There exist constants $K<\infty$ and $\lambda>0$ such that

$$
\mathrm{E} \exp \left(\frac{\lambda \sup _{0 \leq t \leq T}\left\|\int_{0}^{t} e^{A(t-s)} \psi(s) \mathrm{d} W(s)\right\|_{L^{q}(\mu)}^{2}}{\|\| \psi \|\left._{L^{2}\left((0, T) ; \gamma\left(U, L^{q}(\mu)\right)\right)}\right|_{\infty} ^{2}}\right) \leq K
$$


holds for every progressively measurable $\psi \in L^{\infty}\left(\Omega ; L^{2}\left((0, T) ; \gamma\left(U, L^{q}(\mu)\right)\right)\right)$.

In particular, we obtain an exponential tail estimate:

$$
\mathrm{P}\left\{\sup _{0 \leq t \leq T}\left\|\int_{0}^{T} e^{A(t-s)} \psi(s) \mathrm{d} W(s)\right\|_{L^{q}(\mu)} \geq \varepsilon\right\} \leq K \exp \left(-\frac{\lambda \varepsilon^{2}}{\kappa}\right)
$$

for all $\varepsilon>0$ and every process $\psi \in \Gamma\left(U, L^{q}(\mu)\right)$ satisfying

$$
\underset{\Omega}{\operatorname{esssup}} \int_{0}^{T}\|\psi\|_{\gamma\left(U, L^{q}(\mu)\right)}^{2} \leq \kappa .
$$

In this form, the estimate (2.3) seems to be new, but a closely related result was obtained in [5], Theorem 1.3, by a completely different (and more complicated) method based on the Itô formula.

Analogously we may proceed if $\left(e^{A t}\right)$ is a semigroup whose (negative) generator has a bounded $H^{\infty}$-calculus of angle less than $\frac{\pi}{2}$ since then again a suitable dilation theorem is available.

Theorem 2.2. Theorem 2.1 remains true under the following hypotheses: $(M$, $\mathscr{M}, \mu)$ is a separable measure space, $q \in\left[2, \infty\left[, T \in \mathbb{R}_{+}\right.\right.$, and $\left(e^{A t}\right)$ is a $C_{0^{-}}$ semigroup on $L^{q}(\mu)$ with an infinitesimal generator $A$ such that $-A$ has a bounded $H^{\infty}$-calculus of $H^{\infty}$-angle $\omega_{H^{\infty}}(-A)<\frac{\pi}{2}$.

Theorems 2.1 and 2.2 are closely related but independent: $-A$ has a bounded $H^{\infty}$-calculus if $A$ generates a positive contractive $C_{0}$-semigroup on $L^{q}(\mu)$, however, without an analyticity assumption it may happen that $\omega_{H \infty}(-A)>\frac{\pi}{2}$. (Cf. Corollaries 10.15 and 10.16 in [15].) The proof of Theorem 2.2 is deferred to the end of Section 2.

If dilation results are not available, we have to resort to the factorization method (cf. [7], where this method is thoroughly dealt with), which provides $L^{p}$-estimates of stochastic convolutions for $p>2$ without any additional hypotheses on the semigroup. Instead of a Burkholder-type estimate (2.2) one gets, however, only a weaker inequality

$$
\mathrm{E} \sup _{0 \leq t \leq T}\left\|\int_{0}^{t} e^{A(t-s)} \psi(s) \mathrm{d} W(s)\right\|_{X}^{p} \leq D_{p}^{p} \mathrm{E} \int_{0}^{T}\|\psi(s)\|_{\gamma}^{p} \mathrm{~d} s .
$$

If $X$ is a Hilbert space, it was shown in [24], Theorem 2.3, that $D_{p}=O(\sqrt{p}), p \rightarrow \infty$, so from (2.4) exponential $L^{2}$-integrability of stochastic convolutions may be easily inferred. The proof remains essentially the same for 2-smooth Banach spaces $X$, only the Burkholder-Davis-Gundy inequality must be replaced with Theorem 1.1. Let us denote by $\|\cdot\|_{\gamma, \infty}$ the norm of the Banach space $L^{\infty}([0, T] \times \Omega ; \gamma(U, X))$, that is,

$$
\|\psi\|_{\gamma, \infty}=\operatorname{ess~sup}_{(s, \omega) \in[0, T] \times \Omega}\|\psi\|_{\gamma(U, X)} .
$$


Proceeding as in the proof of Theorem 1.1 in [24] we arrive at the following result:

Theorem 2.3. Let $\left(e^{A t}\right)$ be a $C_{0}$-semigroup on a separable 2-smooth Banach space $X$ and $T \in \mathbb{R}_{+}$. There exist constants $K_{1}<\infty$ and $\lambda_{1}>0$ such that

$$
\mathrm{E} \exp \left(\frac{\lambda_{1}}{\|\psi\|_{\gamma, \infty}^{2}} \sup _{0 \leq t \leq T}\left\|\int_{0}^{t} e^{A(t-s)} \psi(s) \mathrm{d} W(s)\right\|_{X}^{2}\right) \leq K_{1}
$$

for every progressively measurable $\psi \in L^{\infty}([0, T] \times \Omega ; \gamma(U, X))$.

Due to Theorem 2.3, exponential tail estimates (2.3) hold for all $\varepsilon>0$ and any process $\psi \in \Gamma(U, X)$ satisfying

$$
\underset{[0, T] \times \Omega}{\operatorname{ess} \sup }\|\psi\|_{\gamma(U, X)}^{2} \leq \kappa
$$

The stochastic convolution $J(\psi)$ is well defined under the assumption (2.1), but all results hitherto considered depended on a more stringent hypothesis $\psi \in \Gamma(U, X)$. Now we show that Theorem 2.3 remains valid for processes which are only $\mathscr{L}(U, X)$ valued, provided the semigroup $\left(e^{A t}\right)$ consists of radonifying operators.

Let us denote by $\mathscr{L}_{s}(U, X)$ the space of all bounded linear operators from $U$ to $X$ endowed with the strong operator topology and by $S_{T, X}^{\infty}$ the space of all progressively measurable mappings $\psi:[0, T] \times \Omega \longrightarrow \mathscr{L}_{s}(U, X)$ such that

$$
[\psi]_{\infty, X}=\operatorname{essip}_{(t, \omega) \in[0, T] \times \Omega}\|\psi(t, \omega)\|_{\mathscr{L}(U, X)}<\infty .
$$

(Let us recall that $\psi$ is progressively measurable as an $\mathscr{L}_{s}(U, X)$-valued process if and only if $\psi u$ is a progressively measurable $X$-valued process for all $u \in U$. Owing to separability, $\|\psi\|_{\mathscr{L}(U, X)}$ is then a real-valued progressively measurable function.)

We shall denote by $\mathfrak{P}_{\gamma}$ the (Banach) operator ideal of $\gamma$-summing operators. (Recall that for Banach spaces $E$ and $F$ the space $\mathfrak{P}_{\gamma}(E, F)$ consists of all $B \in$ $\mathscr{L}(E, F)$ such that

$$
\sup _{n \geq 1} \mathrm{E}\left\|\sum_{k=1}^{n} g_{k} B x_{k}\right\|_{F}^{2}<\infty
$$

for all weakly 2-summable sequences $\left\{x_{i}\right\}$ in $E$ and a sequence $\left\{g_{i}\right\}$ of independent standard Gaussian random variables. If $E$ is a separable Hilbert space and $F$ does not contain a copy of $c_{0}$, then $\mathfrak{P}_{\gamma}(E, F)=\gamma(E, F)$, cf. e.g. [18], Proposition 2.4.)

Mimicking the proof of Theorem 1.3 from [24] we obtain:

Theorem 2.4. Let $\left(e^{A t}\right)$ be a $C_{0}$-semigroup on a separable 2-smooth Banach space $X$ and $T \in \mathbb{R}_{+}$. Let there exist $\left.\gamma \in\right] 0,1[$ such that

$$
\int_{0}^{T} t^{-\gamma}\left\|e^{A t}\right\|_{\mathfrak{P}_{\gamma}(X, X)}^{2} \mathrm{~d} t<\infty
$$


Then there exist constants $K_{2}<\infty$ and $\lambda_{2}>0$ such that

$$
\mathrm{E} \exp \left(\frac{\lambda_{2}}{[\psi]_{\infty, X}^{2}} \sup _{0 \leq t \leq T}\left\|\int_{0}^{t} e^{A(t-s)} \psi(s) \mathrm{d} W(s)\right\|_{X}^{2}\right) \leq K_{2}
$$

holds for all $\psi \in S_{T, X}^{\infty}$.

We shall return to the assumption (2.5) in Section 4, but let us indicate here that the stochastic convolution $J(\psi)$ is well defined under the hypotheses of Theorem 2.4. The space $X$ being reflexive surely does not contain a copy of $c_{0}$, thus

$$
\left\|e^{A(t-s)} \psi_{s}\right\|_{\gamma(U, X)}=\left\|e^{A(t-s)} \psi_{s}\right\|_{\mathfrak{P}_{\gamma}(U, X)} \leq\left\|e^{A(t-s)}\right\|_{\mathfrak{P}_{\gamma}(X, X)}\left\|\psi_{s}\right\|_{\mathscr{L}(U, X)} .
$$

If we suppose that

$$
\int_{0}^{T} \mathrm{E}\left\|\psi_{s}\right\|_{\mathscr{L}(U, X)}^{p} \mathrm{~d} s<\infty
$$

for some $p \geq 2$, then $J_{t}(\psi)$ is well defined for almost all $t \in[0, T]$ and a standard application of the factorization procedure yields that $J(\psi)$ has a continuous modification for $p>2 / \gamma$.

Processes $\psi$ corresponding to diffusion terms in standard stochastic partial differential equations are often only $\mathscr{L}_{s}(U, X)$-valued, but they may take values in $\gamma(U, Y)$ for some superspace $Y$ of $X$. This situation is covered by the following result, the proof of which is almost identical with that of the preceding theorem.

Theorem 2.5. Let $\left(e^{A t}\right)$ be a $C_{0}$-semigroup on a separable 2-smooth Banach space $X$ and $T \in \mathbb{R}_{+}$. Suppose that there exists a separable Banach space $Y$ such that $X$ is embedded in $Y$, the operators $e^{A t}, t>0$, may be extended to operators in $\mathscr{L}(Y, X)$ and

$$
\int_{0}^{T} t^{-\gamma}\left\|e^{A t}\right\|_{\mathscr{L}(Y, X)}^{2} \mathrm{~d} t<\infty
$$

for some $\gamma \in] 0,1\left[\right.$. Then there exist constants $K_{3}<\infty$ and $\lambda_{3}>0$ such that

$$
\mathrm{E} \exp \left(\frac{\lambda_{3} \sup _{0 \leq t \leq T}\left\|\int_{0}^{t} e^{A(t-s)} \psi(s) \mathrm{d} W(s)\right\|_{X}^{2}}{\|\psi\|_{L^{\infty}([0, T] \times \Omega ; \gamma(U, Y))}^{2}}\right) \leq K_{3}
$$

holds for all progressively measurable $\psi \in L^{\infty}([0, T] \times \Omega ; \gamma(U, Y))$.

Remark 2.1. In an important particular case when there exists a $\gamma$-radonifying embedding $j: U \hookrightarrow Y$ we may apply Theorem 2.5 to processes $j \psi$ with $\psi \in S_{T, U}^{\infty}$ obtaining

$$
\mathrm{E} \exp \left(\frac{\lambda_{4}}{[\psi]_{\infty, U}^{2}} \sup _{0 \leq t \leq T}\left\|\int_{0}^{t} e^{A(t-s)} \psi(s) \mathrm{d} W(s)\right\|_{X}^{2}\right) \leq K_{4}
$$


for some $K_{4}<\infty, \lambda_{4}>0$ and any $\psi \in S_{T, U}^{\infty}$.

Other results from [24], as estimates over unbounded time intervals or estimates in norms of interpolation spaces between $X$ and $\operatorname{Dom}(A)$ may extended to 2-smooth spaces in the same way. We shall not dwell on it, since no new ingredients besides Theorem 1.1 are needed.

Proof of Theorem 2.2. Suppose that $(M, \mathscr{M}, \mu)$ is a separable measure space, $q \in\left[2, \infty\left[\right.\right.$, and $\left(e^{A t}\right)$ is a $C_{0}$-semigroup on $L^{q}(\mu)$ with an infinitesimal generator $A$. Assume further that $-A$ has a bounded $H^{\infty}$-calculus of the $H^{\infty}$-angle $\omega_{H \infty}(-A)<\frac{\pi}{2}$. According to Corollary 5.4 in [11] there exist a separable measure space $(N, \mathscr{N}, \nu)$, a closed subspace $Y$ of $L^{q}(\nu)$, an isomorphism $J: L^{q}(\mu) \longrightarrow Y$, a bounded projection $P: L^{q}(\nu) \longrightarrow Y$ and a bounded group $\left(B_{t}\right)$ on $L^{q}(\nu)$ such that the diagram

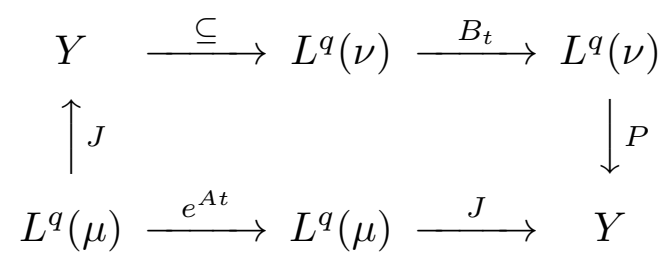

commutes for all $t \geq 0$, that is, $J e^{A t}=P B_{t} J, t \geq 0$. Let us set

$$
\delta=\left\|J^{-1}\right\|_{\mathscr{L}\left(Y, L^{q}(\mu)\right)} \sup _{t \geq 0}\left\|P B_{t}\right\|_{\mathscr{L}\left(L^{q}(\nu), Y\right)}, \quad \zeta=\sup _{t \leq 0}\left\|B_{t} J\right\|_{\mathscr{L}\left(L^{q}(\mu), L^{q}(\nu)\right)}
$$

and take $T \in \mathbb{R}_{+}, p>2$ and $\psi \in \Gamma\left(U, L^{q}(\mu)\right)$ arbitrary. Since $L^{q}(\nu)$ is a separable 2 -smooth space, Theorem 1.1 is applicable and we get

$$
\begin{aligned}
\left.\left|\sup _{0 \leq t \leq T}\left\|\int_{0}^{t} e^{A(t-s)} \psi(s) \mathrm{d} W(s)\right\|\right|_{L^{q}(\mu)}\right|_{p} \\
=\left.\left|\sup _{0 \leq t \leq T}\left\|J^{-1} \int_{0}^{t} J e^{A(t-s)} \psi(s) \mathrm{d} W(s)\right\|\right|_{L^{q}(\mu)}\right|_{p} \\
\leq\left.\left\|J^{-1}\right\| \sup _{0 \leq t \leq T}\left\|\int_{0}^{t} J e^{A(t-s)} \psi(s) \mathrm{d} W(s)\right\|_{Y}\right|_{p} \\
=\left\|J^{-1}\right\|\left|\sup _{0 \leq t \leq T}\left\|\int_{0}^{t} P B_{t-s} J \psi(s) \mathrm{d} W(s)\right\|\right|_{Y} \\
\leq\left\|J^{-1}\right\|\left|\sup _{0 \leq t \leq T}\left\|P B_{t}\right\| \mathscr{L}\left(L^{q}(\nu), Y\right)\left\|\int_{0}^{t} B B_{-s} J \psi(s) \mathrm{d} W(s)\right\|_{L^{q}(\nu)}\right|_{p} \\
\leq \delta\left|\sup _{0 \leq t \leq T}\left\|\int_{0}^{t} B B_{-s} J \psi(s) \mathrm{d} W(s)\right\| \|_{L^{q}(\nu)}\right|_{p} \mid \\
\leq \delta \Pi \sqrt{p}\left|\left(\int_{0}^{T}\left\|B_{-s} J \psi(s)\right\|_{\gamma\left(U, L^{q}(\nu)\right)}^{2} \mathrm{~d} s\right)^{1 / 2}\right|_{p}
\end{aligned}
$$


Let $\left\{g_{n}\right\}$ be a sequence of independent standard Gaussian random variables and $\left\{k_{n}\right\}$ an orthonormal basis of $U$. By the definition of the norm in $\gamma\left(U, L^{q}(\nu)\right)$ we have

$$
\begin{aligned}
\left\|B_{-s} J \psi(s)\right\|_{\gamma\left(U, L^{q}(\nu)\right)}^{2} & =\mathrm{E}\left\|\sum_{n} g_{n} B_{-s} J \psi(s) k_{n}\right\|_{L^{q}(\nu)}^{2}=\mathrm{E}\left\|B_{-s} J \sum_{n} g_{n} \psi(s) k_{n}\right\|_{L^{q}(\nu)}^{2} \\
& \leq \zeta^{2} \mathrm{E}\left\|\sum_{n} g_{n} \psi(s) k_{n}\right\|_{L^{q}(\mu)}^{2}=\zeta^{2}\|\psi(s)\|_{\gamma\left(U, L^{q}(\mu)\right)}^{2} .
\end{aligned}
$$

Whence we obtain that

$$
\left|\sup _{0 \leq t \leq T}\left\|\int_{0}^{t} e^{A(t-s)} \psi(s) \mathrm{d} W(s)\right\|_{L^{q}(\mu)}\right|_{p} \leq \delta \zeta \Pi \sqrt{p}\left|\left(\int_{0}^{T}\|\psi(s)\|_{\gamma\left(U, L^{q}(\mu)\right)}^{2} \mathrm{~d} s\right)^{1 / 2}\right|_{p}
$$

holds for every $T>0, p>2$ and $\psi \in \Gamma\left(U, L^{q}(\mu)\right)$ and thus the extrapolation theorem may be used exactly in the same manner as in the proof of Theorem 2.1. Q.E.D.

3. Proof of Theorem 1.1. We begin with a well known lemma on approximation with simple processes. For reader's convenience, we shall sketch its proof since only the case $p=2$ is treated explicitly in [18]. Let us recall that a process in $\Gamma(U, X)$ is called simple, if it is of the form

$$
\psi(s, \omega)=\sum_{i=0}^{n-1} \sum_{j=1}^{m} \mathbf{1}_{F_{i j}}(\omega) A_{i j} \mathbf{1}_{] t_{i}, t_{i+1}\right]}(s), \quad 0 \leq s \leq T, \omega \in \Omega,
$$

where $\varrho=\left\{0=t_{0}<t_{1}<\cdots<t_{n}=T\right\}$ is a partition of the interval $[0, T]$, $A_{i j} \in \operatorname{Fin}(\Upsilon, X), i=0, \ldots, n-1, j=1, \ldots, m$, are finite dimensional operators, and $\left\{F_{i j}, 1 \leq j \leq m\right\} \subseteq \mathscr{F}_{t_{i}}$ is a system of disjoint sets for each $i=0, \ldots, n-1$.

Lemma 3.1. Let $p \geq 2, T>0$, and let $\psi \in \Gamma(U, X)$ be such that

$$
\mathrm{E}\left(\int_{0}^{T}\|\psi(s)\|_{\gamma}^{2} \mathrm{~d} s\right)^{p / 2}<\infty
$$

Then a sequence $\left\{\psi_{n}\right\}_{n \geq 1}$ of simple processes may be found such that

$$
\lim _{n \rightarrow \infty} \mathrm{E}\left(\int_{0}^{T}\left\|\psi_{n}(s)-\psi(s)\right\|_{\gamma}^{2} \mathrm{~d} s\right)^{p / 2}=0
$$

Proof. Lemma 3.4 from [18] shown that there exists a sequence $F_{n}: \gamma(U, X) \longrightarrow$ $\operatorname{Fin}(\Upsilon, X), n \geq 1$, of Borel mappings satisfying $\left\|F_{n}(y)-y\right\|_{\gamma} \searrow 0$ as $n \rightarrow \infty$ for all $y \in \gamma(U, X), \operatorname{Rng} F_{n}$ having cardinality $n$ for all $n \geq 1$. In particular, Rng $F_{1}=\{Z\}$ for some $Z \in \operatorname{Fin}(\Upsilon, X)$. Since

$$
\left\|F_{n}(y)-y\right\|_{\gamma}^{2} \leq\left\|F_{1}(y)-y\right\|_{\gamma}^{2} \leq 2\|Z\|_{\gamma}^{2}+2\|y\|_{\gamma}^{2}, \quad y \in \gamma(U, X),
$$


the dominated convergence theorem implies that

$$
\int_{0}^{T}\left\|F_{n}(\psi(s))-\psi(s)\right\|_{\gamma}^{2} \mathrm{~d} s \underset{n \rightarrow \infty}{\longrightarrow} 0 \quad \text { P-almost surely }
$$

hence also

$$
\mathrm{E}\left(\int_{0}^{T}\left\|F_{n}(\psi(s))-\psi(s)\right\|_{\gamma}^{2} \mathrm{~d} s\right)^{p / 2} \underset{n \rightarrow \infty}{\longrightarrow} 0 .
$$

Obviously, for any $n$ fixed we can find progressively measurable sets $M_{1}, \ldots, M_{r}$ and operators $A_{1}, \ldots, A_{r} \in \operatorname{Fin}(\Upsilon, U)$ such that

$$
F_{n}(\psi(s, \omega))=\sum_{i=1}^{r} A_{i} \mathbf{1}_{M_{i}}(s, \omega), \quad s \in[0, T], \omega \in \Omega .
$$

A standard result yields real-valued simple processes $m_{i, k}$ satisfying

$$
\lim _{k \rightarrow \infty} \mathrm{E} \int_{0}^{T}\left|\mathbf{1}_{M_{i}}(s)-m_{i, k}(s)\right|^{p} \mathrm{~d} s=0
$$

and the proof of Lemma 3.1 may be completed easily. Q.E.D.

Proof of Theorem 1.1. As usual, it suffices to prove (1.1) only for finite deterministic times $\tau=T \in \mathbb{R}_{+}$. Indeed, assume that the estimate

$$
\left|\sup _{0 \leq t \leq T}\left\|I_{t}(\psi)\right\|_{X}\right|_{p} \leq \Pi \sqrt{p}\left|\left(\int_{0}^{T}\|\psi(s)\|_{\gamma}^{2} \mathrm{~d} s\right)^{1 / 2}\right|_{p}
$$

has been established for all $T>0$. Then (1.1) holds for $\tau=+\infty$ by the monotone convergence theorem and for any stopping time $\lambda$ we have

$$
\sup _{0 \leq t \leq \lambda}\left\|I_{t}(\psi)\right\|_{X}=\sup _{t \geq 0}\left\|I_{t \wedge \lambda}(\psi)\right\|_{X}=\sup _{t \geq 0}\left\|I_{t}\left(\mathbf{1}_{[0, \lambda[} \psi\right)\right\|_{X}
$$

(cf. [18], Lemma 3.6), which proves our claim.

So, let us fix $p>2$ and $T>0$ arbitrarily. First, we shall prove (1.1) for simple processes. Suppose that $\psi$ is a simple process of the form (3.1) for some partition $\varrho=\left\{0=t_{0}<t_{1}<\cdots<t_{n}=T\right\}$ of the interval $[0, T]$. We shall denote by

$$
\boldsymbol{n}(\varrho)=\max _{0 \leq i \leq n-1}\left|t_{i+1}-t_{i}\right|
$$

mesh of the partition $\varrho$. The estimate of the $p$-th moment of $I_{T}(\psi)$ will be deduced from an estimate for discrete time martingales due to I. Pinelis. Towards this end, let us set

$$
f_{0}=0, \quad f_{k}=\sum_{i=0}^{k-1} \sum_{j=1}^{m} \mathbf{1}_{F_{i j}} A_{i j}\left(W\left(t_{i+1}\right)-W\left(t_{i}\right)\right), \quad 1 \leq k \leq n,
$$


and note that $f_{n}=I_{T}(\psi)$. Further, set $d_{k}=f_{k}-f_{k-1}, 1 \leq k \leq n$, and $\mathfrak{f}_{i}=\mathscr{F}_{t_{i}}$, $0 \leq i \leq n$. Then $\left(f_{k}, \mathfrak{f}_{k}\right)$ is a martingale in $X$, let us denote by

$$
s_{n}(f)=\left(\sum_{k=1}^{n} \mathrm{E}\left(\left\|d_{k}\right\|_{X}^{2} \mid \mathfrak{f}_{k-1}\right)\right)^{1 / 2}
$$

its conditional square function. According to [22], Theorem 4.1, we have

$$
\left|\max _{1 \leq k \leq n}\left\|f_{k}\right\|_{X}\right|_{p} \leq \Lambda p\left|\max _{1 \leq k \leq n}\left\|d_{k}\right\|_{X}\right|_{p}+\Lambda \sqrt{p}\left|s_{n}(f)\right|_{p}
$$

for a constant $\Lambda$ depending only on $\left(X,\|\cdot\|_{X}\right)$ (hence, in particular, independent of both $p$ and $\left.\left(f_{k}\right)\right)$. With our choice of $\left(f_{k}\right)$, let us estimate the terms on the right hand side of (3.2). First,

$$
\begin{aligned}
\left|s_{n}(f)\right|_{p}^{p} & =\mathrm{E}\left(\sum_{k=0}^{n-1} \mathrm{E}\left(\left\|d_{k+1}\right\|_{X}^{2} \mid \mathfrak{f}_{k}\right)\right)^{p / 2} \\
& =\mathrm{E}\left(\sum_{k=0}^{n-1} \mathrm{E}\left(\left\|\sum_{j=1}^{m} \mathbf{1}_{F_{k j}} A_{k j}\left(W\left(t_{k+1}\right)-W\left(t_{k}\right)\right)\right\|^{2} \mid \mathscr{F}_{t_{k}}\right)\right)^{p / 2} \\
& =\mathrm{E}\left(\sum_{k=0}^{n-1} \mathrm{E}\left(\sum_{j=1}^{m} \mathbf{1}_{F_{k j}}\left\|A_{k j}\left(W\left(t_{k+1}\right)-W\left(t_{k}\right)\right)\right\|^{2} \mid \mathscr{F}_{t_{k}}\right)\right)^{p / 2} \\
& =\mathrm{E}\left(\sum_{k=0}^{n-1} \sum_{j=1}^{m} \mathbf{1}_{F_{k j}} \mathrm{E}\left(\left\|A_{k j}\left(W\left(t_{k+1}\right)-W\left(t_{k}\right)\right)\right\|^{2} \mid \mathscr{F}_{t_{k}}\right)\right)^{p / 2} \\
& =\mathrm{E}\left(\sum_{k=0}^{n-1} \sum_{j=1}^{m} \mathbf{1}_{F_{k j}} \mathrm{E}\left\|A_{k j}\left(W\left(t_{k+1}\right)-W\left(t_{k}\right)\right)\right\|^{2}\right)^{p / 2} \\
& =\mathrm{E}\left(\sum_{k=0}^{n-1} \sum_{j=1}^{m} \mathbf{1}_{F_{k j}}\left\|A_{k j} Q^{1 / 2}\right\|_{\gamma(\Upsilon, X)}^{2}\left(t_{k+1}-t_{k}\right)\right)^{p / 2} \\
& =\mathrm{E}\left(\int_{0}^{T}\|\psi(s)\|_{\gamma}^{2} \mathrm{~d} s\right)^{p / 2},
\end{aligned}
$$

where we used disjointness of $F_{k 1}, \ldots, F_{k m}$ in the third equality and independence of increments of $A_{k j} W$ in the fifth one.

Secondly,

$$
\begin{aligned}
\left.\max _{1 \leq k \leq n}\left\|d_{k}\right\|_{X}\right|_{p} ^{p} & =\mathrm{E}\left(\max _{0 \leq k \leq n-1}\left\|d_{k+1}\right\|\right)^{p}=\mathrm{E} \max _{0 \leq k \leq n-1}\left\|d_{k+1}\right\|_{X}^{p} \\
& \leq \sum_{k=0}^{n-1} \mathrm{E}\left\|d_{k+1}\right\|_{X}^{p}
\end{aligned}
$$




$$
\begin{aligned}
& =\sum_{k=0}^{n-1} \mathrm{E}\left\|\sum_{j=1}^{m} \mathbf{1}_{F_{k j}} A_{k j}\left(W\left(t_{k+1}\right)-W\left(t_{k}\right)\right)\right\|^{p} \\
= & \sum_{k=0}^{n-1} \sum_{j=1}^{m} \mathrm{E}\left\{\mathbf{1}_{F_{k j}}\left\|A_{k j}\left(W\left(t_{k+1}\right)-W\left(t_{k}\right)\right)\right\|^{p}\right\} \\
= & \sum_{k=0}^{n-1} \sum_{j=1}^{m} \mathrm{P}\left(F_{k j}\right) \mathrm{E}\left\|A_{k j}\left(W\left(t_{k+1}\right)-W\left(t_{k}\right)\right)\right\|^{p} \\
\leq & \left.\alpha_{p}^{p} \sum_{k=0}^{n-1} \sum_{j=1}^{m}\left(t_{k+1}-t_{k}\right)^{p / 2} \mathrm{P}\left(F_{k j}\right)\left\|A_{k j} Q^{1 / 2}\right\|_{\gamma(\Upsilon, X)}^{p}\right) \\
\leq & \alpha_{p}^{p} \boldsymbol{n}(\varrho)^{\frac{p}{2}-1} \mathrm{E} \sum_{k=0}^{n-1} \sum_{j=1}^{m} \mathbf{1}_{F_{k j}}\left\|A_{k j} Q^{1 / 2}\right\|_{\gamma(\Upsilon, X)}^{p}\left(t_{k+1}-t_{k}\right) \\
= & \alpha_{p}^{p} \boldsymbol{n}(\varrho)^{\frac{p}{2}-1} \mathrm{E} \int_{0}^{T}\left\|\psi_{s}\right\|_{\gamma}^{p} \mathrm{~d} s .
\end{aligned}
$$

Therefore, we get

$$
\left|\left\|I_{T}(\psi)\right\|_{X}\right|_{p} \leq \Lambda \alpha_{p} p \boldsymbol{n}(\varrho)^{\frac{1}{2}-\frac{1}{p}}\left|\int_{0}^{T}\left\|\psi_{s}\right\|_{\gamma}^{p} \mathrm{~d} s\right|_{1}^{1 / p}+\Lambda \sqrt{p}\left|\left(\int_{0}^{T}\left\|\psi_{s}\right\|_{\gamma}^{2} \mathrm{~d} s\right)^{1 / 2}\right|_{p} .
$$

Let $\sigma=\left\{0=s_{0}<s_{1}<\cdots<s_{N}=T\right\}$ be a partition of $[0, T]$ finer than $\varrho$, that is, there exist $r(i)$ such that $s_{r(i)}=t_{i}, i=0, \ldots, n$. Let us define a simple function $\tilde{\psi}$ by a formula

$$
\tilde{\psi}(s, \omega)=\sum_{r=0}^{N-1} \sum_{j=1}^{m} \mathbf{1}_{H_{r j}}(\omega) \tilde{A}_{r j} \mathbf{1}_{]_{r}, s_{r+1}\right]}(s), \quad 0 \leq s \leq T, \omega \in \Omega
$$

where $H_{r j}=F_{i j}, \tilde{A}_{r j}=A_{i j}$ for $r(i) \leq r<r(i+1), i=0, \ldots, n-1$. Repeating the proof of (3.3) we obtain

$$
\left|\left\|I_{T}(\tilde{\psi})\right\|_{X}\right|_{p} \leq \Lambda \alpha_{p} p \boldsymbol{n}(\sigma)^{\frac{1}{2}-\frac{1}{p}}\left|\int_{0}^{T}\left\|\tilde{\psi}_{s}\right\|_{\gamma}^{p} \mathrm{~d} s\right|_{1}^{1 / p}+\Lambda \sqrt{p}\left|\left(\int_{0}^{T}\left\|\tilde{\psi}_{s}\right\|_{\gamma}^{2} \mathrm{~d} s\right)^{1 / 2}\right|_{p} .
$$

However, we plainly have

$$
I_{T}(\psi)=I_{T}(\tilde{\psi}), \quad \int_{0}^{T}\left\|\psi_{s}\right\|_{\gamma}^{q} \mathrm{~d} s=\int_{0}^{T}\|\tilde{\psi}\|_{\gamma}^{q} \mathrm{~d} s \quad \text { P-almost surely }
$$

for each $q \geq 2$ by the very definition of $\tilde{\psi}$, so

$$
\left|\left\|I_{T}(\psi)\right\|_{X}\right|_{p} \leq \Lambda p \alpha_{p} \boldsymbol{n}(\sigma)^{\frac{1}{2}-\frac{1}{p}}\left|\int_{0}^{T}\left\|\psi_{s}\right\|_{\gamma}^{p} \mathrm{~d} s\right|_{1}^{1 / p}+\Lambda \sqrt{p}\left|\left(\int_{0}^{T}\left\|\psi_{s}\right\|_{\gamma}^{2} \mathrm{~d} s\right)^{1 / 2}\right|_{p} .
$$


For any $\varepsilon>0$ we may choose a partition $\sigma$ with mesh $\boldsymbol{n}(\sigma)<\varepsilon$, thus (3.4) implies

$$
||\left|I_{T}(\psi) \|_{X}\right|_{p} \leq \Lambda \sqrt{p}\left|\left(\int_{0}^{T}\left\|\psi_{s}\right\|_{\gamma}^{2} \mathrm{~d} s\right)^{1 / 2}\right|_{p} .
$$

The process $\left(\left\|I_{\bullet}(\psi)\right\|_{X}\right)$ is a continuous submartingale, hence from the Doob inequality we get

$$
\begin{aligned}
\left|\sup _{0 \leq t \leq T}\left\|I_{t}(\psi)\right\|_{X}\right|_{p} & \leq\left(\frac{p}{p-1}\right)\left|\left\|I_{T}(\psi)\right\|_{X}\right|_{p} \\
& \leq 2 \Lambda \sqrt{p}\left|\left(\int_{0}^{T}\|\psi\|_{\gamma}^{2} \mathrm{~d} s\right)^{1 / 2}\right|_{p}
\end{aligned}
$$

Setting $\Pi=2 \Lambda$ we conclude that Theorem 1.1 holds true for simple processes $\psi$.

Finally, take $\psi \in \Gamma(U, X)$ arbitrary. We may assume that

$$
\mathrm{E}\left(\int_{0}^{T}\|\psi(s)\|_{\gamma}^{2} \mathrm{~d} s\right)^{p / 2}<\infty
$$

since otherwise there is nothing to prove. By Lemma 3.1 there exist simple processes $\psi_{n}, n \in \mathbb{N}$, such that

$$
\lim _{n \rightarrow \infty} \mathrm{E}\left(\int_{0}^{T}\left\|\psi_{n}(s)-\psi(s)\right\|_{\gamma}^{2} \mathrm{~d} s\right)^{p / 2}=0
$$

consequently,

$$
\lim _{n \rightarrow \infty} \mathrm{E}\left(\int_{0}^{T}\left\|\psi_{n}(s)\right\|_{\gamma}^{2} \mathrm{~d} s\right)^{p / 2}=\mathrm{E}\left(\int_{0}^{T}\|\psi(s)\|_{\gamma}^{2} \mathrm{~d} s\right)^{p / 2} .
$$

The construction of a stochastic integral using a Burkholder-type inequality for $p=2$, as presented in [18], $\S 3$, implies that, by passing to a subsequence if necessary, we may obtain

$$
\sup _{0 \leq t \leq T}\left\|I_{t}\left(\psi_{n}\right)\right\|_{X} \underset{n \rightarrow \infty}{\longrightarrow} \sup _{0 \leq t \leq T}\left\|I_{t}(\psi)\right\|_{X} \quad \text { P-almost surely }
$$

from (3.5). Hence the Fatou lemma yields

$$
\begin{aligned}
\mathrm{E} \sup _{0 \leq t \leq T}\left\|I_{t}(\psi)\right\|_{X}^{p} & \leq \liminf _{n \rightarrow \infty} \mathrm{E} \sup _{0 \leq t \leq T}\left\|I_{t}\left(\psi_{n}\right)\right\|_{X}^{p} \\
& \leq \Pi^{p} p^{p / 2} \liminf _{n \rightarrow \infty} \mathrm{E}\left(\int_{0}^{T}\left\|\psi_{n}(s)\right\|_{\gamma}^{2} \mathrm{~d} s\right)^{p / 2} \\
& =\Pi^{p} p^{p / 2} \mathrm{E}\left(\int_{0}^{T}\|\psi(s)\|_{\gamma}^{2} \mathrm{~d} s\right)^{p / 2}
\end{aligned}
$$


and the proof of Theorem 1.1 follows. Q.E.D.

4. A note on Theorems 2.4 And 2.5. Now we turn to a simple example illustrating sufficient conditions for applicability of Theorems 2.4 and 2.5. Such conditions are essentially well known and may be found in literature on stochastic partial differential equations, nevertheless we hope that our argument, based on the operator ideals theory, may be of independent interest and may provide some new insights.

First, let us consider the assumption (2.5). If $\left(e^{A t}\right)$ is a bounded analytic semigroup on a Banach space $X$ such that 0 belongs to the resolvent set of its generator, then from [2], Corollary $4.2(\mathrm{~b})$, we know that $\left\|e^{A t}\right\|_{\mathfrak{P}_{\gamma}(X, X)}=O\left(t^{-\theta}\right), t \rightarrow 0+$, provided that $(-A)^{-\theta} \in \mathfrak{P}_{\gamma}(X, X)$. Hence to check $(2.5)$ in this case it suffices to find $\theta \in] 0, \frac{1}{2}\left[\right.$ such that $(-A)^{-\theta} \in \mathfrak{P}_{\gamma}(X, X)$.

Let $G \subseteq \mathbb{R}^{n}$ be a bounded domain with a smooth boundary, set $X=L^{q}(G)$ for some $q \geq 2$ and

$$
\operatorname{Dom}(A)=W_{0}^{1, q}(G) \cap W^{2, q}(G), \quad A u=\Delta u \text { for } u \in \operatorname{Dom}(A) .
$$

As $(-A)^{-\theta}: X \longrightarrow \operatorname{Dom}\left((-A)^{\theta}\right)$ is an isomorphism and $\operatorname{Dom}\left((-A)^{\theta}\right) \subseteq W^{2 \theta, q}(G)$, we get (2.5) if the embedding $W^{2 \theta, q}(G) \hookrightarrow L^{q}(G)$ is $\gamma$-summing.

For $u, v \in[1, \infty]$ and an arbitrary operator ideal $\mathfrak{A}$ set

$$
\sigma_{G}(\mathfrak{A}, u, v)=\inf \left\{\lambda>0 ; \iota \in \mathfrak{A}\left(W^{\lambda, u}(G), L^{v}(G)\right)\right\},
$$

where $W^{\lambda, u}$ are the usual Sobolev-Slobodeckii spaces and $\iota: W^{\lambda, u}(G) \hookrightarrow L^{v}(G)$ is the embedding operator (which is well defined for $\lambda>n\left(\frac{1}{u}-\frac{1}{v}\right) \geq 0$ ). H. König proved (see [21], Theorem 22.7.4) that

$$
\frac{1}{n} \sigma_{G}(\mathfrak{A}, u, v)=\lambda(\mathfrak{A}, u, v)+\frac{1}{u}-\frac{1}{v},
$$

whenever $\mathfrak{A}$ is a quasi-Banach operator ideal and $\lambda(\mathfrak{A}, u, v)$ denotes the limit order of $\mathfrak{A}$ (cf. [21], $\S 14.4$ for the definition of the limit order). By [16], Theorem 8, or [20], Proposition 3.1, if $u, v \in[2, \infty]$ then

$$
\lambda\left(\mathfrak{P}_{\gamma}, u, v\right)=\frac{1}{v} .
$$

Therefore, the embedding $W^{2 \theta, q}(G) \hookrightarrow L^{q}(G)$ is $\gamma$-summing if

$$
2 \theta>n\left(\lambda\left(\mathfrak{P}_{\gamma}, q, q\right)+\frac{1}{q}-\frac{1}{q}\right)=\frac{n}{q} .
$$

We see that, for our choice of $X$ and $A$, the assumption (2.5) is satisfied provided $q>n$.

In applications to stochastic partial differential equations, usually $\Upsilon=L^{2}(G)$ and $\psi$ corresponds to a multiplication operator on $U$. Such operators may belong 
to $\mathscr{L}\left(U, L^{q}(G)\right)$ if the covariance operator $Q$ is "nice", e.g. $\operatorname{Rng} Q^{1 / 2} \subseteq L^{\infty}(G)$, but if the driving process is a standard cylindrical Wiener process one has $U=\Upsilon=$ $L^{2}(G)$ and even bounded diffusion coefficients define multiplication operators only on $L^{2}(G)$. In this case, one may try to apply Theorem 2.5 (or better, Remark 2.1).

Let $Y$ be the completion of $X=L^{q}(G)$ with respect to the norm $\left\|(-A)^{-\theta} \bullet\right\|_{X}$ for some $\theta \in] 0,1 / 2\left[\right.$, then (2.6) is satisfied. Theorem 2.5 will be applicable if $L^{2}(G)$ is embedded into $Y$ and the embedding operator in $\gamma$-radonifying.

For an operator ideal $\mathfrak{A}$ let us denote by $\mathfrak{A}^{\text {dual }}$ its dual ideal, that is

$$
\mathfrak{A}^{\text {dual }}(E, F)=\left\{T \in \mathscr{L}(E, F) ; T^{*} \in \mathfrak{A}\left(F^{*}, E^{*}\right)\right\} .
$$

By [21], Proposition 14.4.7,

$$
\lambda\left(\mathfrak{A}^{\text {dual }}, u, v\right)=\lambda\left(\mathfrak{A}, v^{*}, u^{*}\right)
$$

holds for any operator ideal $\mathfrak{A}$ and $u, v \in[1, \infty]$, where $u^{*}, v^{*}$ denote the dual exponents.

Since $Y=W^{-2 \theta, q}(G)$, the embedding $L^{2}(G) \hookrightarrow Y$ is $\gamma$-summing provided that the embedding $W^{2 \theta, q^{*}}(G) \hookrightarrow L^{2}(G)$ belongs to $\mathfrak{P}_{\gamma}^{\text {dual }}$, which by König's theorem holds true if

$$
\begin{aligned}
2 \theta & >n\left(\lambda\left(\mathfrak{P}_{\gamma}^{\text {dual }}, q^{*}, 2\right)+\frac{1}{q^{*}}-\frac{1}{2}\right) \\
& =n\left(\lambda\left(\mathfrak{P}_{\gamma}, 2, q\right)+\frac{1}{q^{*}}-\frac{1}{2}\right) \\
& =n\left(\frac{1}{q}+\frac{1}{q^{*}}-\frac{1}{2}\right) \\
& =\frac{n}{2} .
\end{aligned}
$$

Therefore, if $X=L^{q}(G)$ for some $q \geq 2$ and $A$ is defined by (4.1), Theorem 2.5 is applicable provided $n=1$.

A note added in proof. This paper having been submitted, I got acquainted with a preprint [25] where the problem of finding an optimal constant in (0.5) is addressed in the particular case $X=L^{q}$ from a very different point of view.

\section{References}

[1] M. T. Barlow, M. Yor: Semi-martingale inequalities via the Garsia-RodemichRumsey lemma, and applications to local times, J. Funct. Anal. 49(1982), 198229

[2] S. Blunck, L. Weis: Operator theoretic properties of semigroups in terms of their generators, Studia Math. 146(2001), 35-54

[3] Z. Brzeźniak: On stochastic convolution in Banach spaces and applications, Stochastics Stochastics Rep. 61(1997), 245-295 
[4] Z. Brzeźniak: Some remarks on Itô and Statonovich integration in 2-smooth Banach spaces, Probabilistic methods in fluids, 48-69, World Sci. Publishing, River Edge 2003

[5] Z. Brzeźniak, S. Peszat: Maximal inequalities and exponential estimates for stochastic convolutions in Banach spaces, Stochastic processes, physics and geometry: new interplays I (Leipzig, 1999), 55-64, Amer. Math. Soc., Providence 2000

[6] P.-L. Chow: Stochastic partial differential equations, Chapman \& Hall/CRC, Boca Raton 2007

[7] G. Da Prato, J. Zabczyk: Stochastic equations in infinite dimensions, Cambridge University Press, Cambridge 1992

[8] B. Davis: On the $\mathrm{L}^{p}$ norms of stochastic integrals and other martingales, Duke Math. J. 43(1976), 697-704

[9] E. Dettweiler: Stochastic integral equations and diffusions on Banach spaces, Probability theory on vector spaces III (Lublin, 1983), 9-45, Lecture Notes in Math. 1080, Springer, Berlin 1984

[10] E. Dettweiler: Stochastic integration relative to Brownian motion on a general Banach space, Doğa Mat. 15(1991), 58-97

[11] A. M. Fröhlich, L. Weis: $H^{\infty}$ calculus and dilations, Bull. Soc. Math. France 134 (2006), 487-508

[12] E. Hausenblas, J. Seidler: Stochastic convolutions driven by martingales: Maximal inequalities and exponential integrability, Stoch. Anal. Appl. 26(2008), 98119

[13] P. Hitczenko: On the behavior of the constant in a decoupling inequality for martingales, Proc. Amer. Math. Soc. 121(1994), 253-258

[14] O. Kallenberg, R. Sztencel: Some dimension-free features of vector-valued martingales, Probab. Theory Related Fields 88(1991), 215-247

[15] P. C. Kunstmann, L. Weis: Maximal $L_{p}$-regularity for parabolic equations, Fourier multiplier theorems and $H^{\infty}$-functional calculus, Functional analytic methods for evolution equations, 65-311, Lecture Notes in Math. 1855, Springer, Berlin 2004

[16] В. Линде, А. Пич: Отображения гауссовских мер цилиндрических множеств в банаховых пространствах, Теор. Вероятност. и Применен. 19 (1974), 472-487 (W. Linde, A. Pitsch: Mappings of Gaussian measures of cylindrical sets in Banach spaces, Teor. Veroyatnost. i Primenen. 19(1974), 472-487

[17] A. L. Neidhardt: Stochastic integrals in 2-uniformly smooth Banach spaces, Ph.D. Thesis, University of Wisconsin 1978

[18] M. Ondreját: Uniqueness for stochastic evolution equations in Banach spaces, Dissertationes Math. 426(2004), 1-63

[19] M. Ondreját: personal communication

[20] C. Michels: $\Lambda(p)$-sets and the limit order of operator ideals, Math. Nachr. 239240(2002), 170-176

[21] A. Pietsch: Operator ideals, Deutscher Verlag der Wissenschaften, Berlin 1978

[22] I. Pinelis: Optimum bounds for the distributions of martingales in Banach spaces, Ann. Probab. 22(1994), 1679-1706; Correction: ibid. 27(1999), 2119 
[23] Y.-F. Ren, H.-Y. Liang: On the best constant in Marcinkiewicz-Zygmund inequality, Statist. Probab. Lett. 53(2001), 227-233

[24] J. Seidler, T. Sobukawa: Exponential integrability of stochastic convolutions, J. London Math. Soc. (2) 67(2003), 245-258

[25] M. Veraar, L. Weis: A note on maximal estimates for stochastic convolutions, preprint arXiv: 1004.5061

[26] A. Zygmund: Trigonometric series, Vol. II, Cambridge University Press, Cambridge 1959 